\title{
Decisions under uncertainty
}

A bioeconomic approach to managing invasive species in Alaska:

The case of Elodea in Chena Slough, Fairbanks

Tobias Schwörer

CNIPM Conference Kodiak October 312012

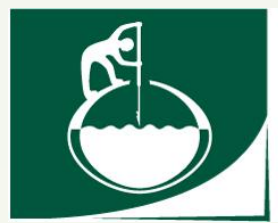

UAA Institute of Social and Economic Research

UNIVERSITY of ALASKA ANCHORAGE 


\section{Outline}

- Ecological economics

- New partnerships

- Valuation of ecosystem services

- Decision and risk analysis: the case of Elodea in Fairbanks

- Is it worth taking action?

- If so, what to do and how much to invest?

- Limitations

- Extensions 


\section{From Greek: Oĩkoৎ "house"}

"The economy is a wholly owned subsidiary of the environment, not the reverse."

Ecosystem and Economy

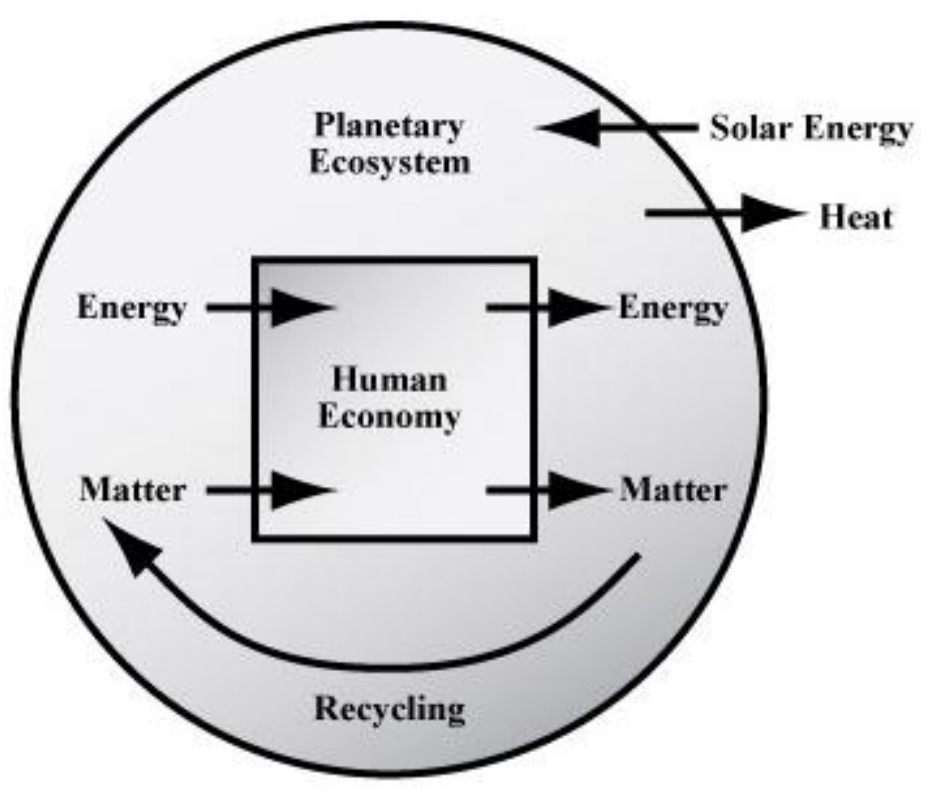

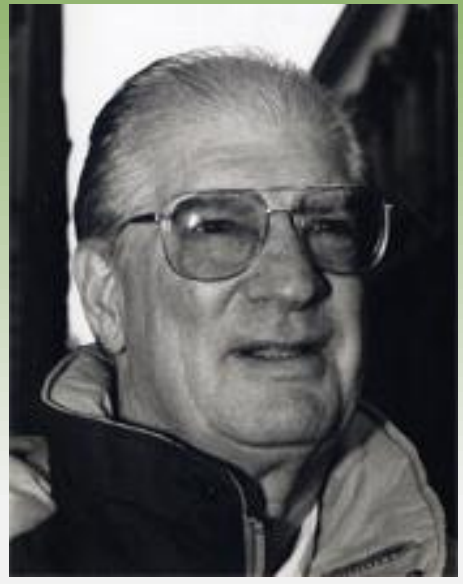

Herman E. Daly 


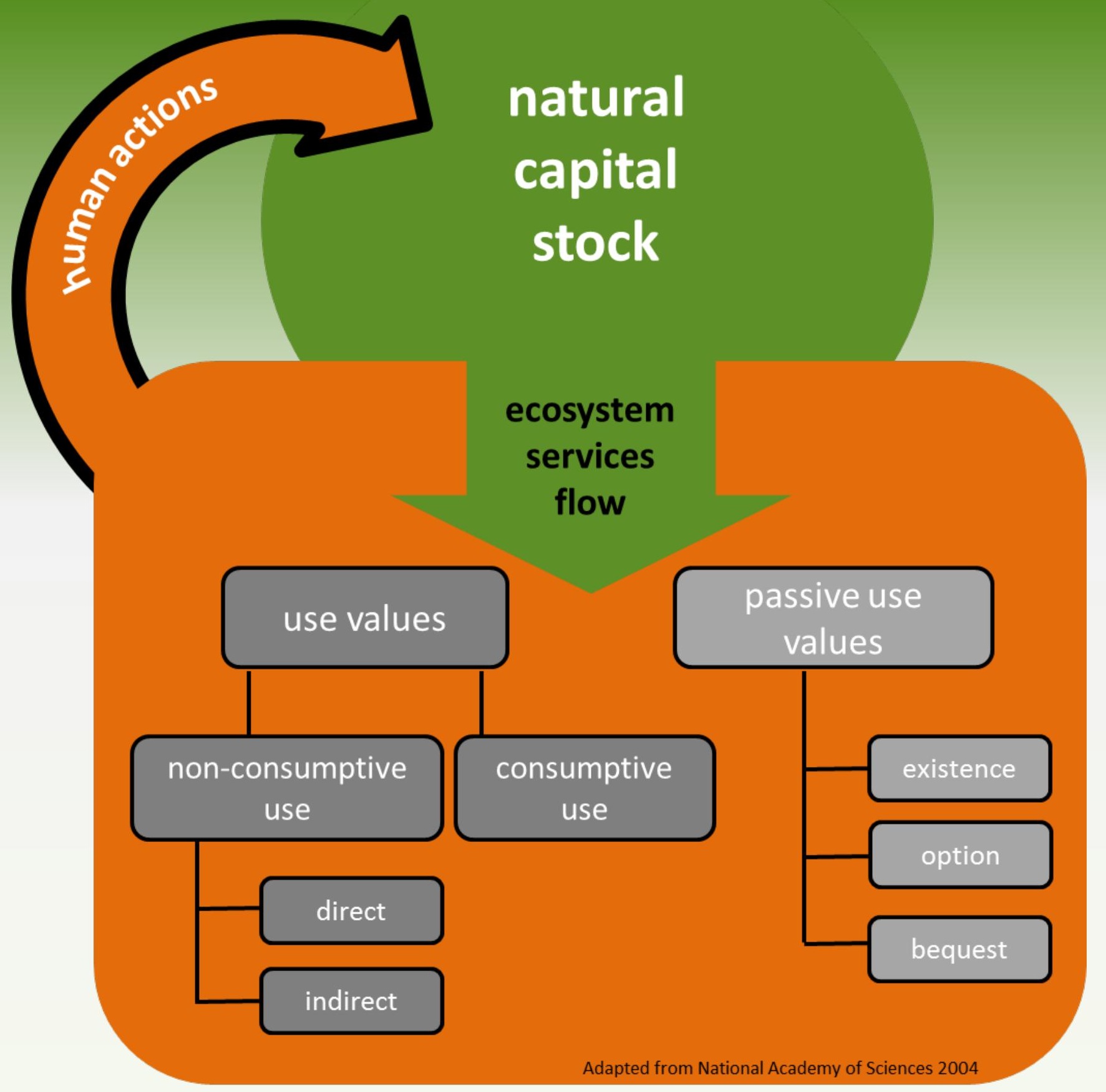




\section{Ecosystem Services}

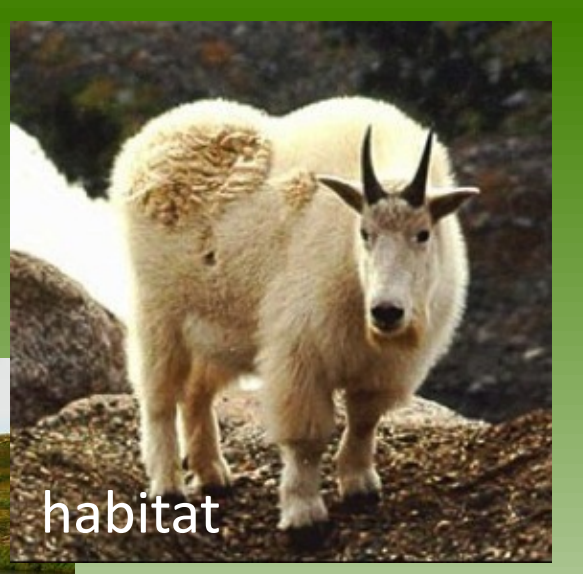

flood protection
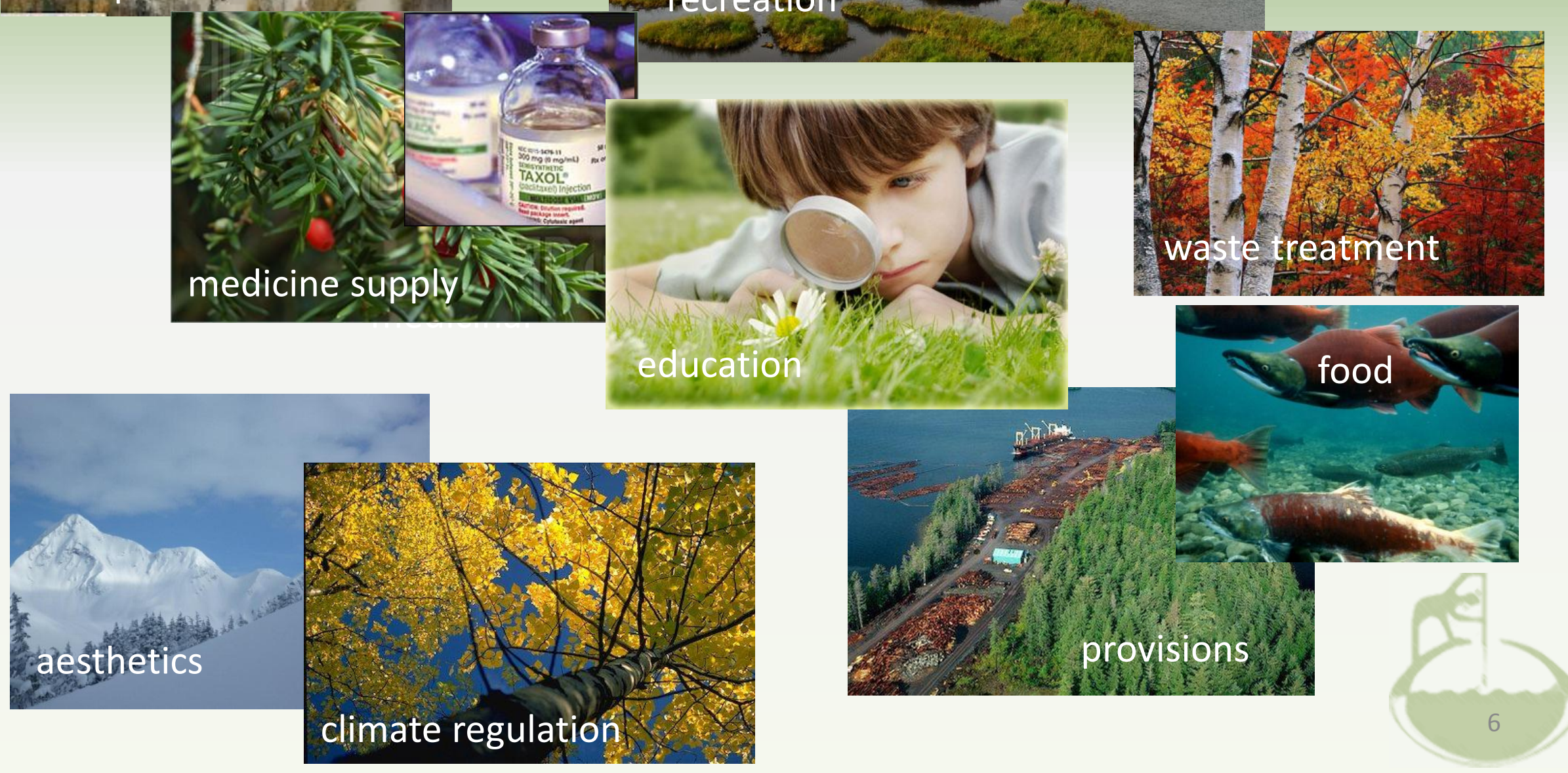


\section{How does this relate to Elodea?}

- Appraisal of damages

- Costs of management actions

- Estimation of expected avoided damages give mgmt. action 

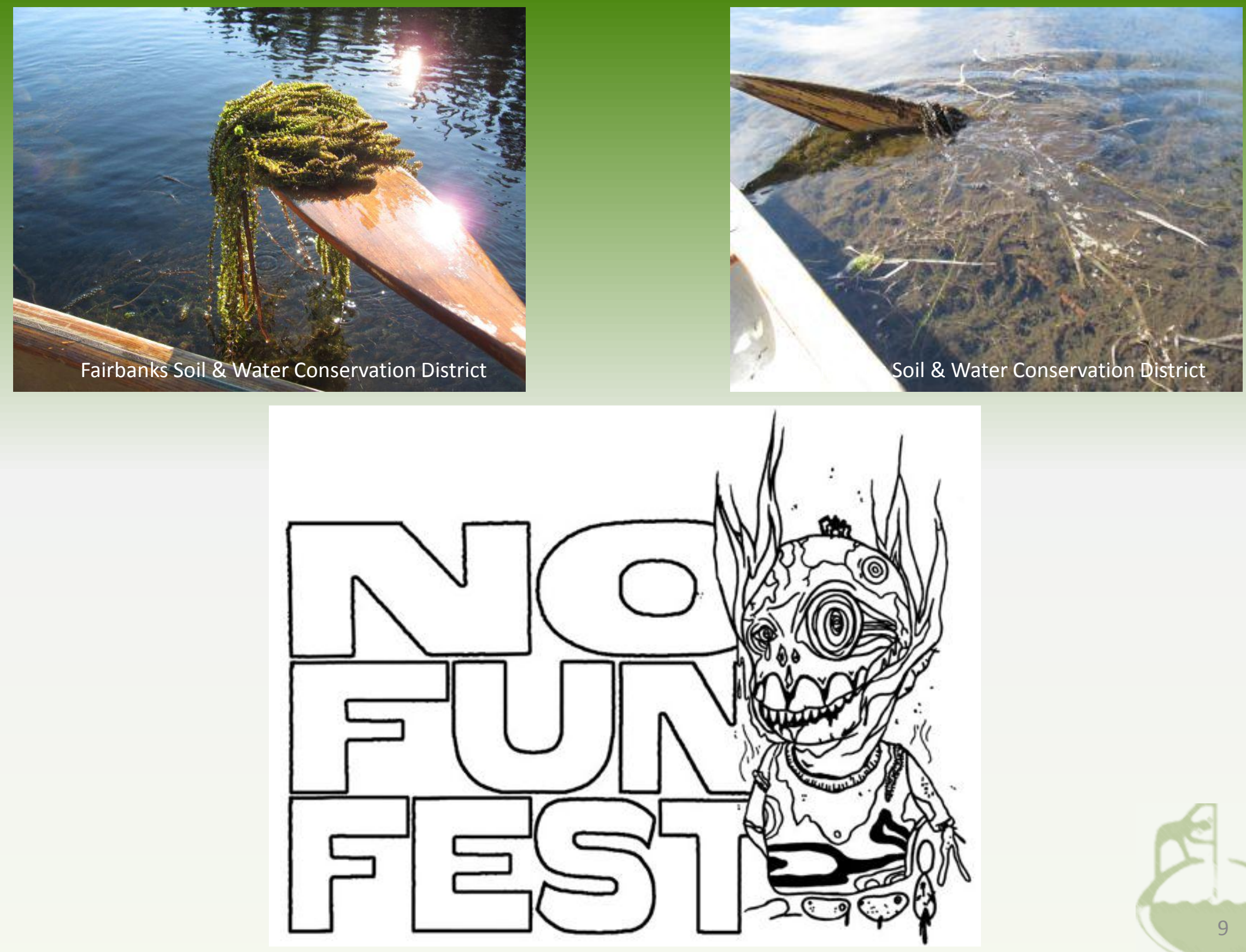


\section{Parameter assumptions}

- Damage values:

- Grayling: \$1.01/ $\mathrm{m}^{2}$ annually

(Duffield et al. 2001)

- Property: $\$ 0.46 / \mathrm{m}^{2}$ annually

(Zhang\&Boyle 2010)

- Canoeing: $\$ 0.13 / \mathrm{m}^{2}$ annually

- Total: $\quad \$ 1.61 / \mathrm{m}^{2}$ annually

(Loomis 2005)

- Management options:

- Suction dredging: $\$ 1.39-\$ 2.46 / \mathrm{m}^{2}$

- Herbicides:

$\$ 0.12-\$ 0.28 / \mathrm{m}^{2}$

(incl. permitting costs $13 \%$ to $100 \%$ of treatment cost)

- Monitoring: $\quad \$ 0.28 / \mathrm{m}^{2}$

- Discount rate: $\quad 4 \%$

- Time horizon: 100 yrs

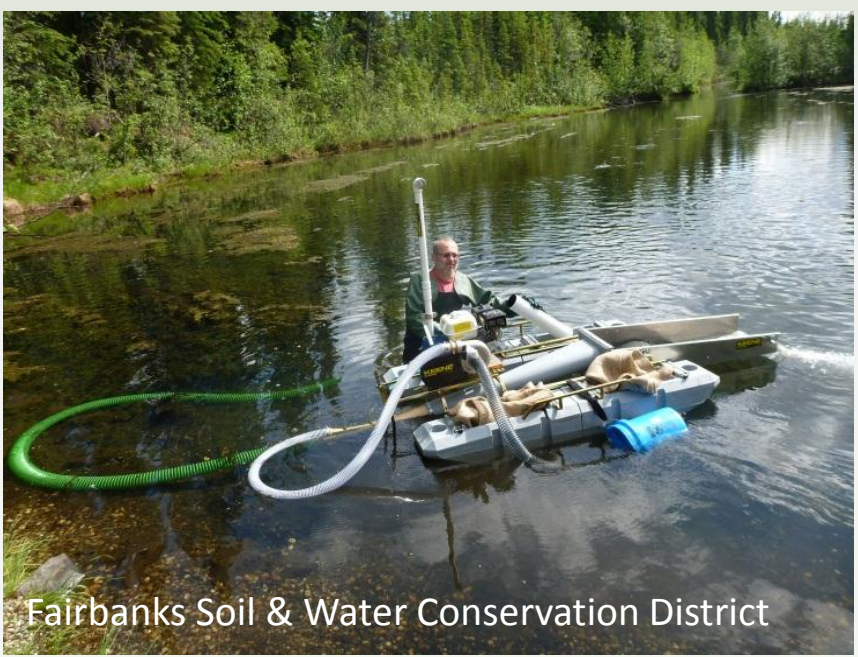




\section{Management Options}

- OPTION A - do nothing

- OPTION B - treat to do something

- OPTION C - treat to maximize benefits

- Decision criteria: NPV greater than zero!

$$
N P V=\sum_{t=1}^{100} \frac{\left(B_{t}-C_{t}\right)}{(1+d)^{t}}
$$

- Results shown levelized

$$
N P V_{\text {levelized }}=N P V\left(\frac{d}{1-(1+d)^{T}}\right)
$$

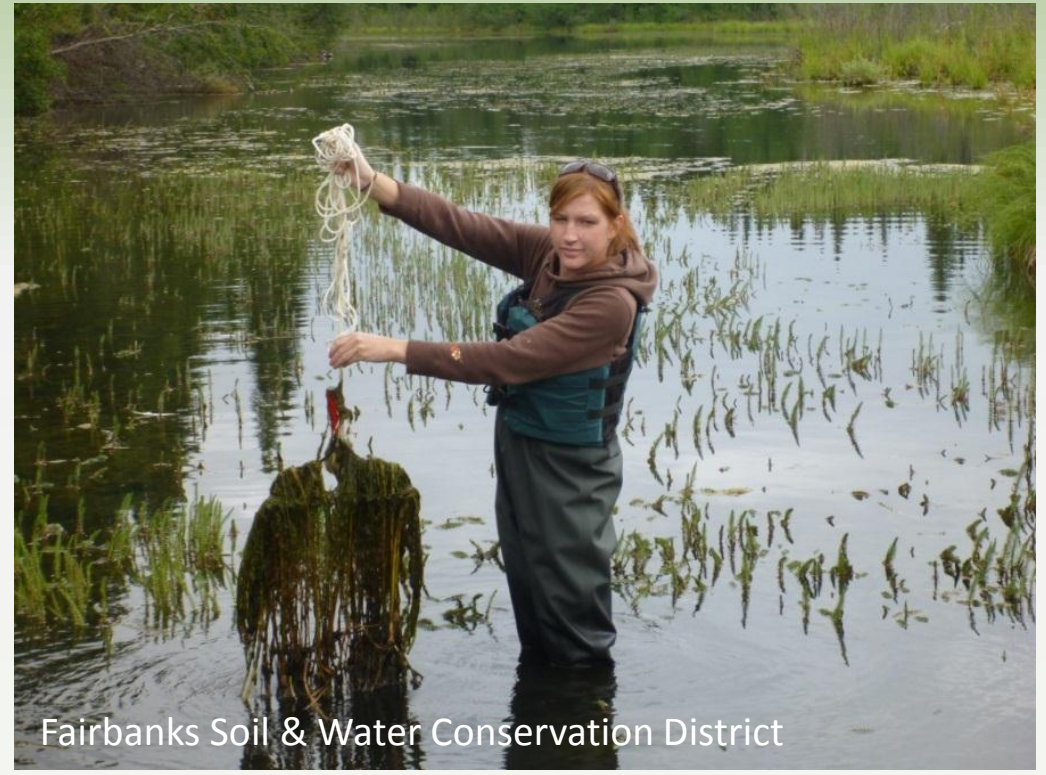




\section{Ecological model}

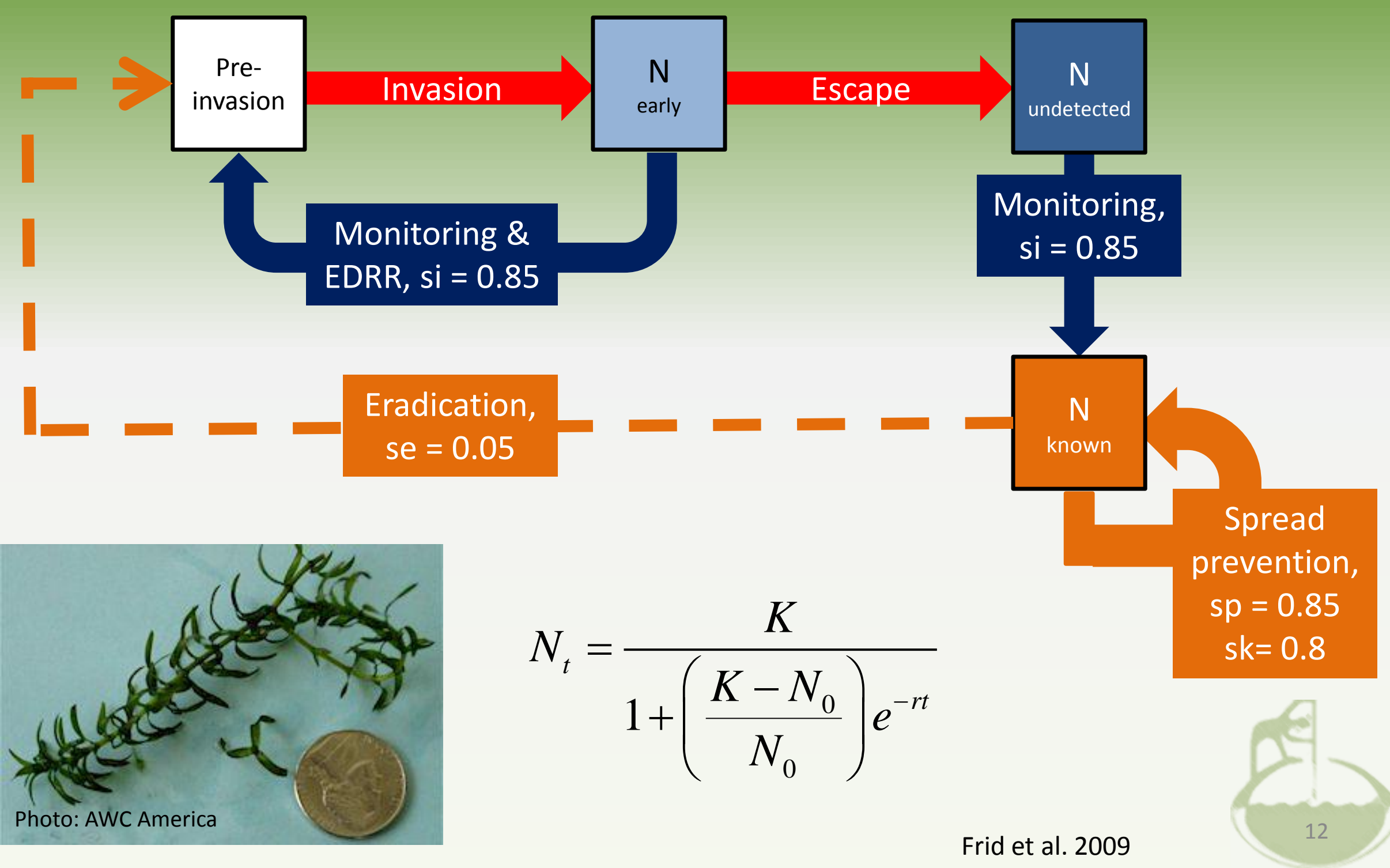




\section{Simulating different ecological outcomes}

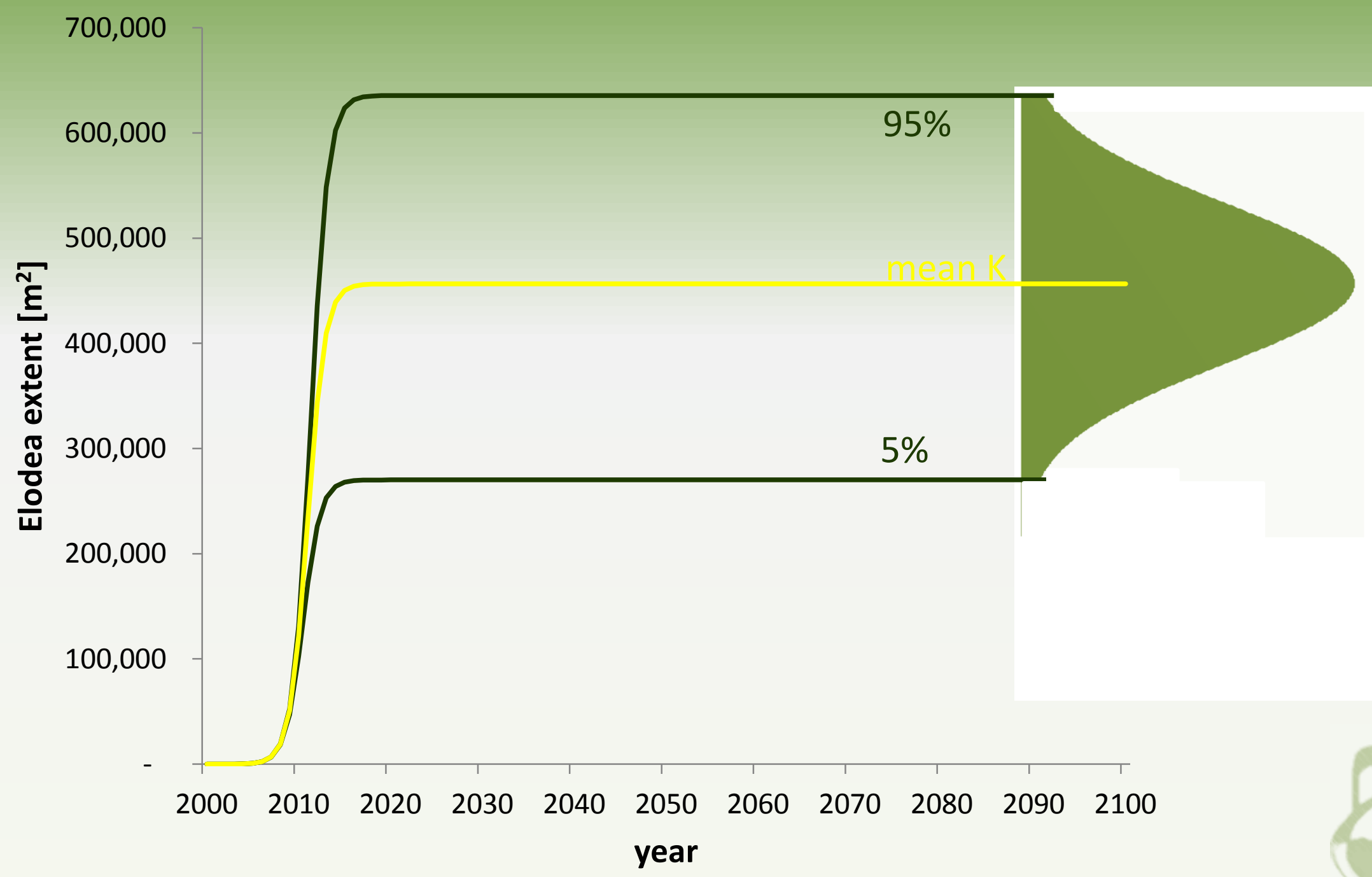




\section{Quantitative Risk and Decision Analysis}

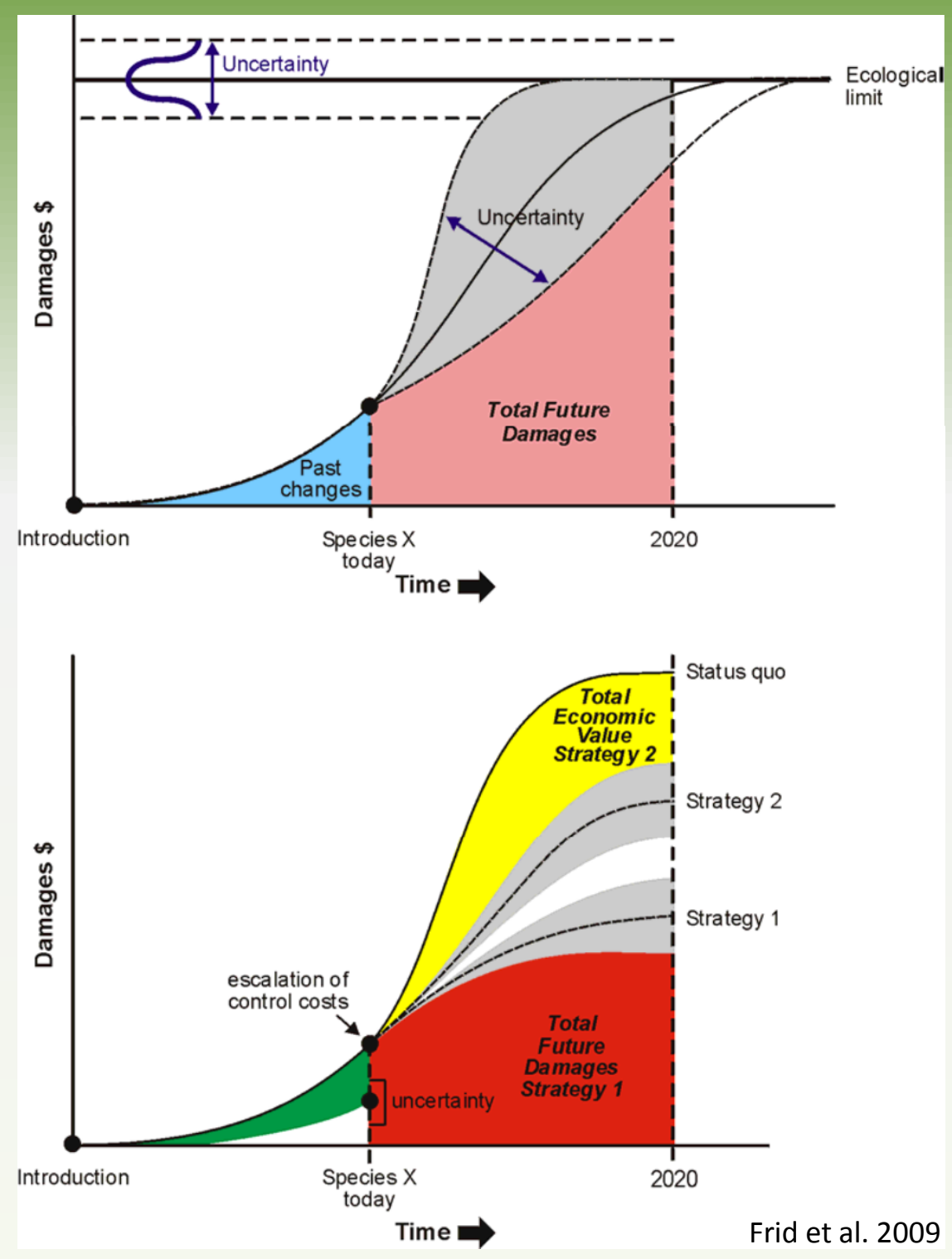

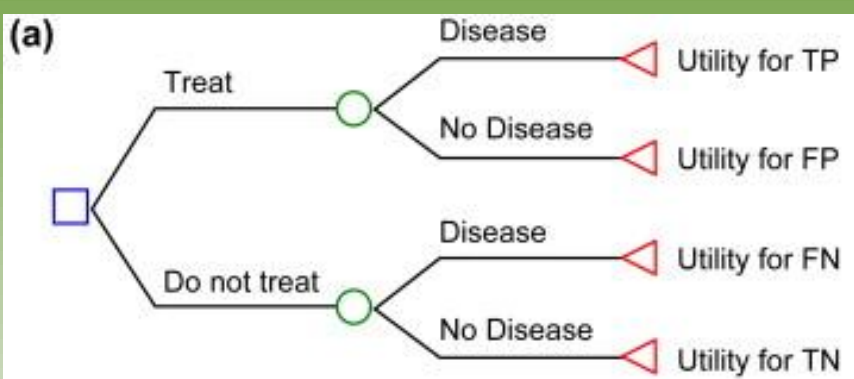

(b)

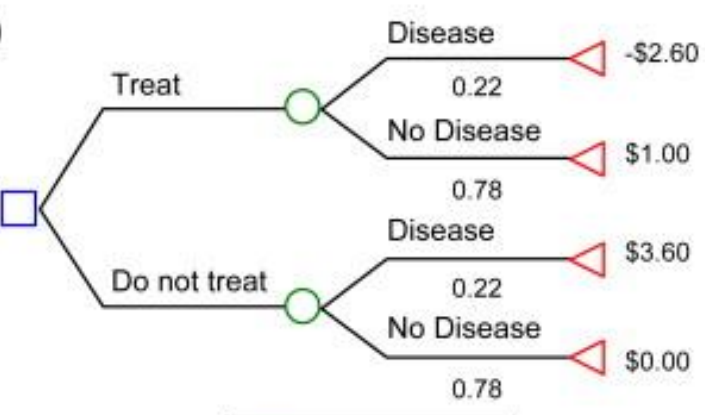

McClamroch et al. 2008 


\section{Simulation Results}

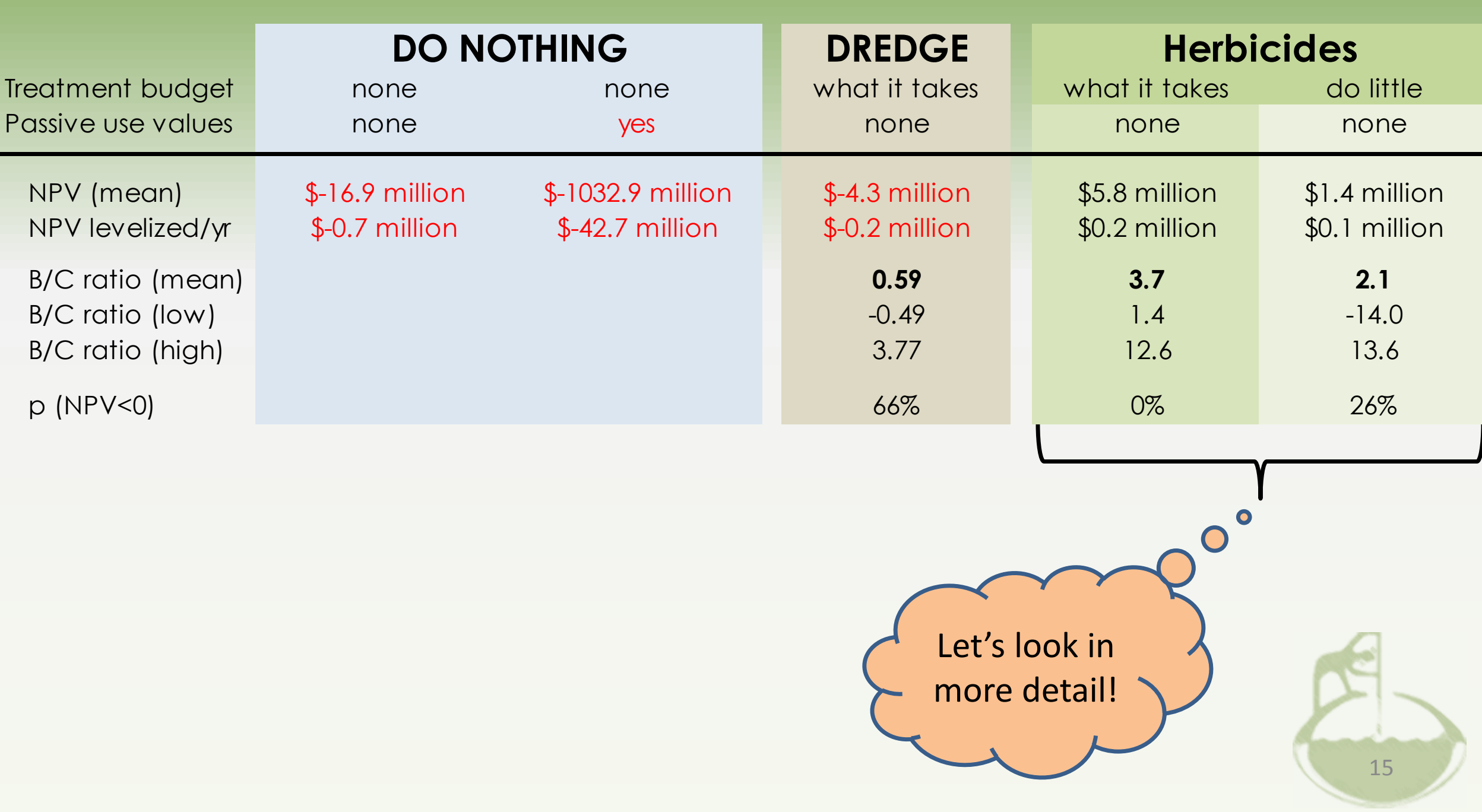




\section{Treat insufficiently $\$ 70,000 /$ year}
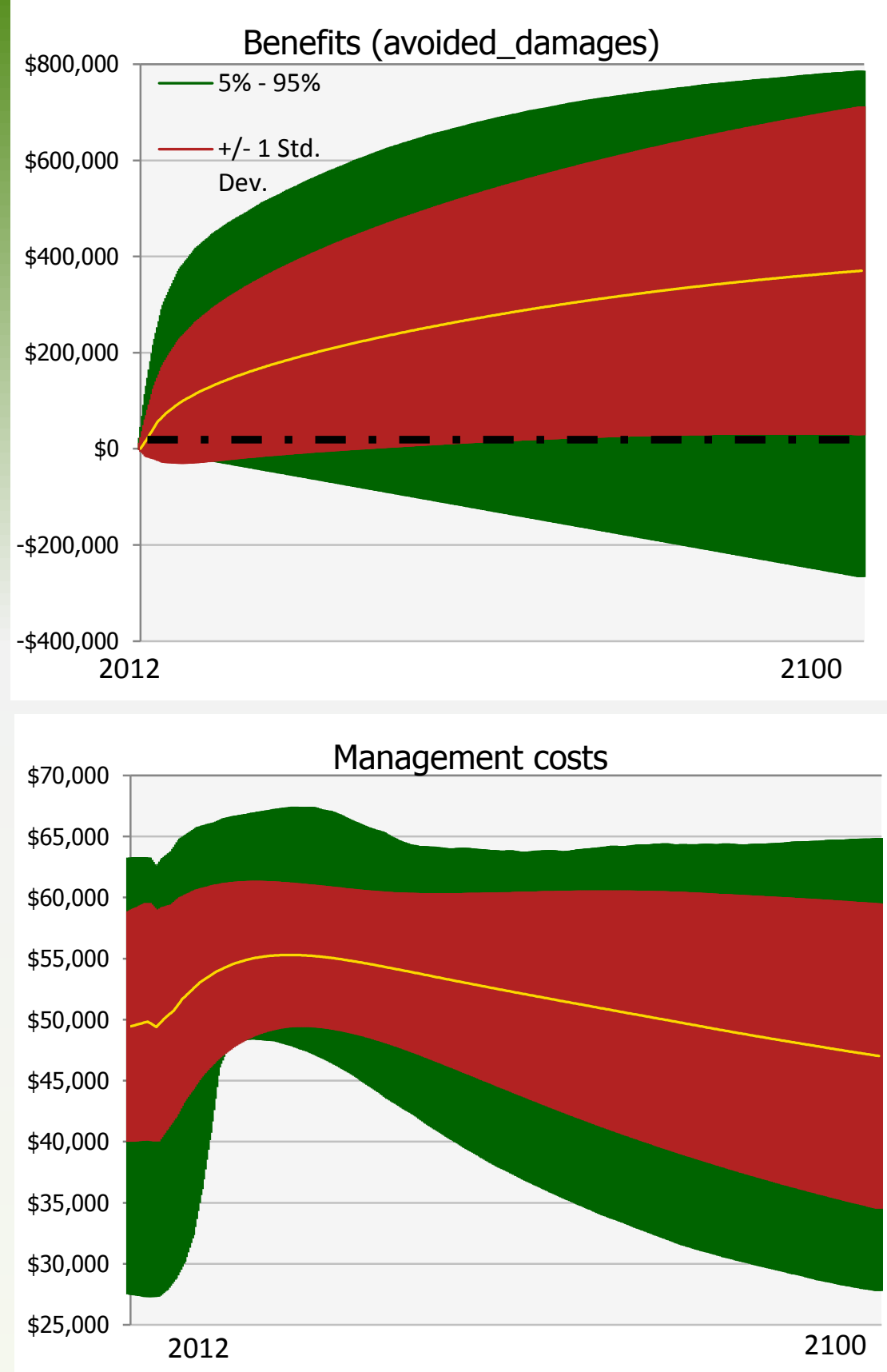

\section{Treat sufficiently $\$ 180,000 /$ year}
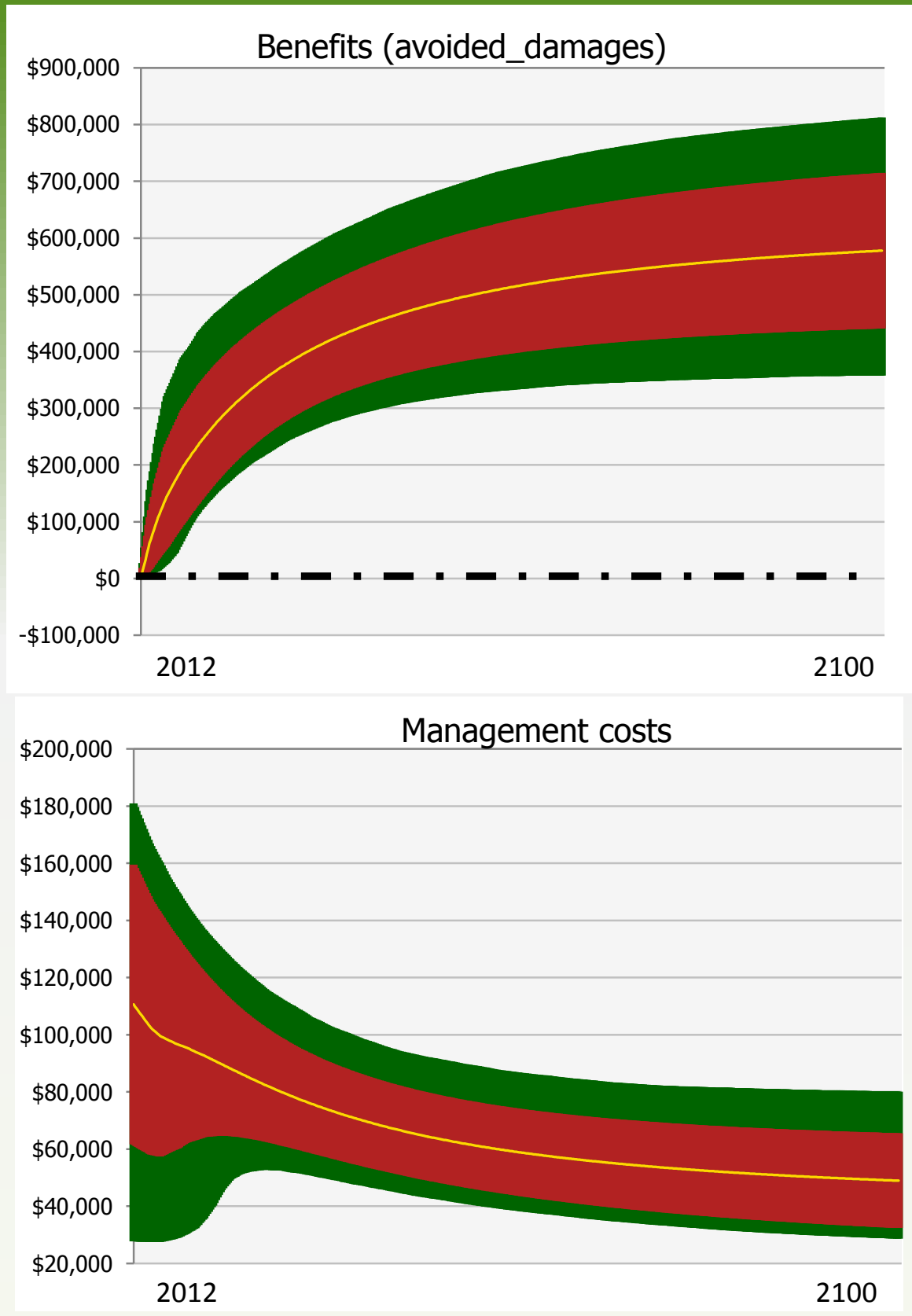


\section{Limitations}

- Actual values of ecosystem services much higher than estimated

- Model only as good as assumptions

- Economic-ecological-linkages

- Unintended consequences

- Risk for non-target organisms

- Data intensive

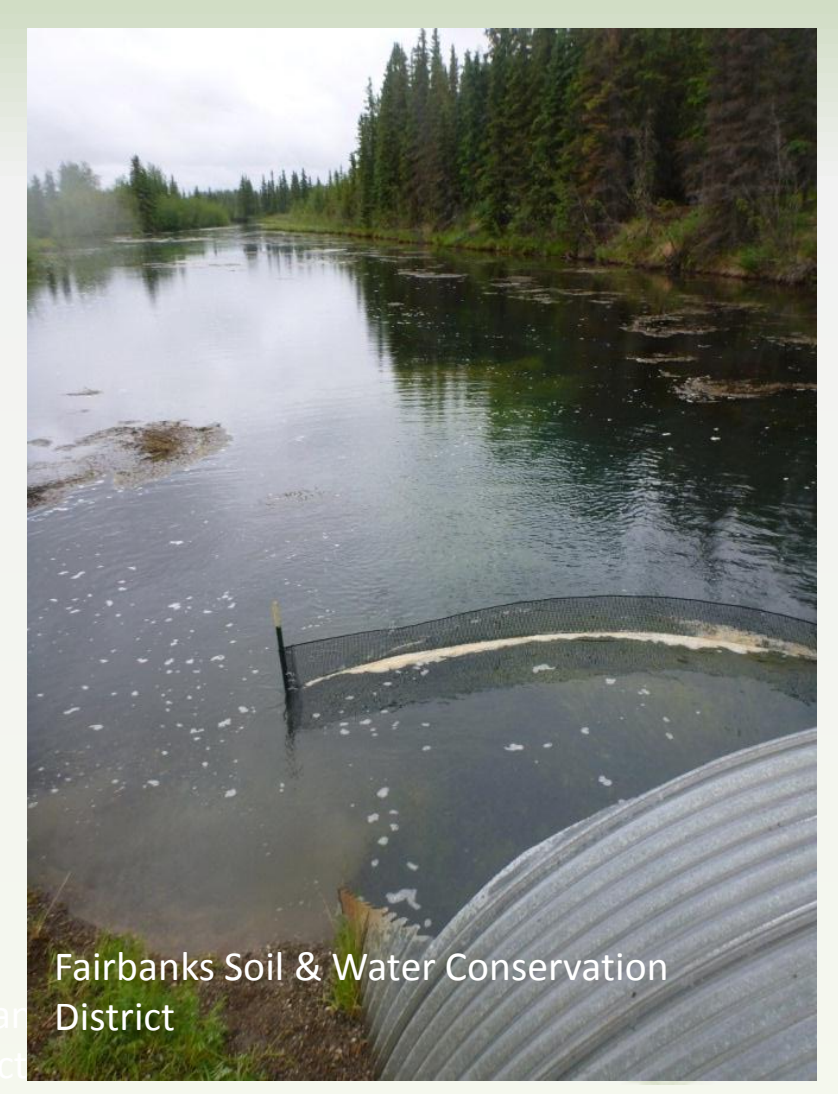




\section{Extensions}

- Additional decision making tool

- times of tight budgets

- D. vex, RCG

- Quantify opportunity cost of delayed action

- Scenario: Elodea crash

(Simpson 1984)

- Logistic growth w/ lag effect

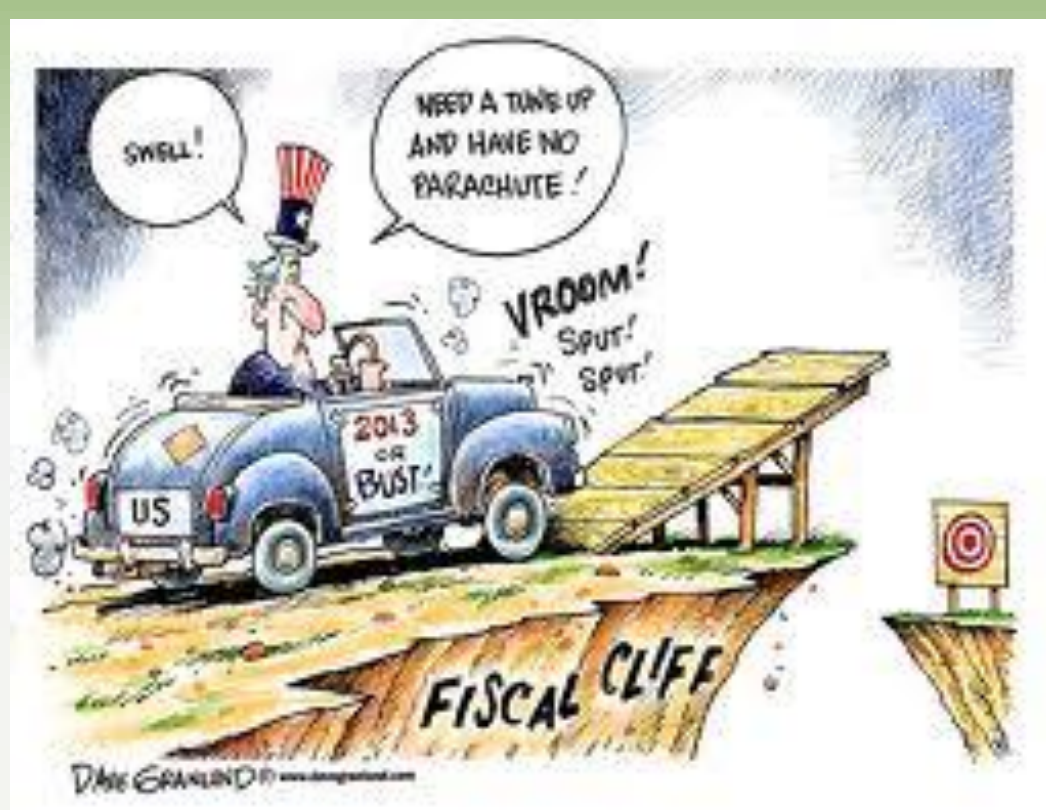

- Biocontrol agents 


\section{Thank you}

- Darcy Etcheverry

Fairbanks Cooperative Weed Management Area

- Nick Lisuzzo, USDA F.S.

- Amy Larson, NPS

- Cecil Rich, USFWS

- Matt Carlson, UAA

- Lindsey Flagstad, UAA

- Many more ... 


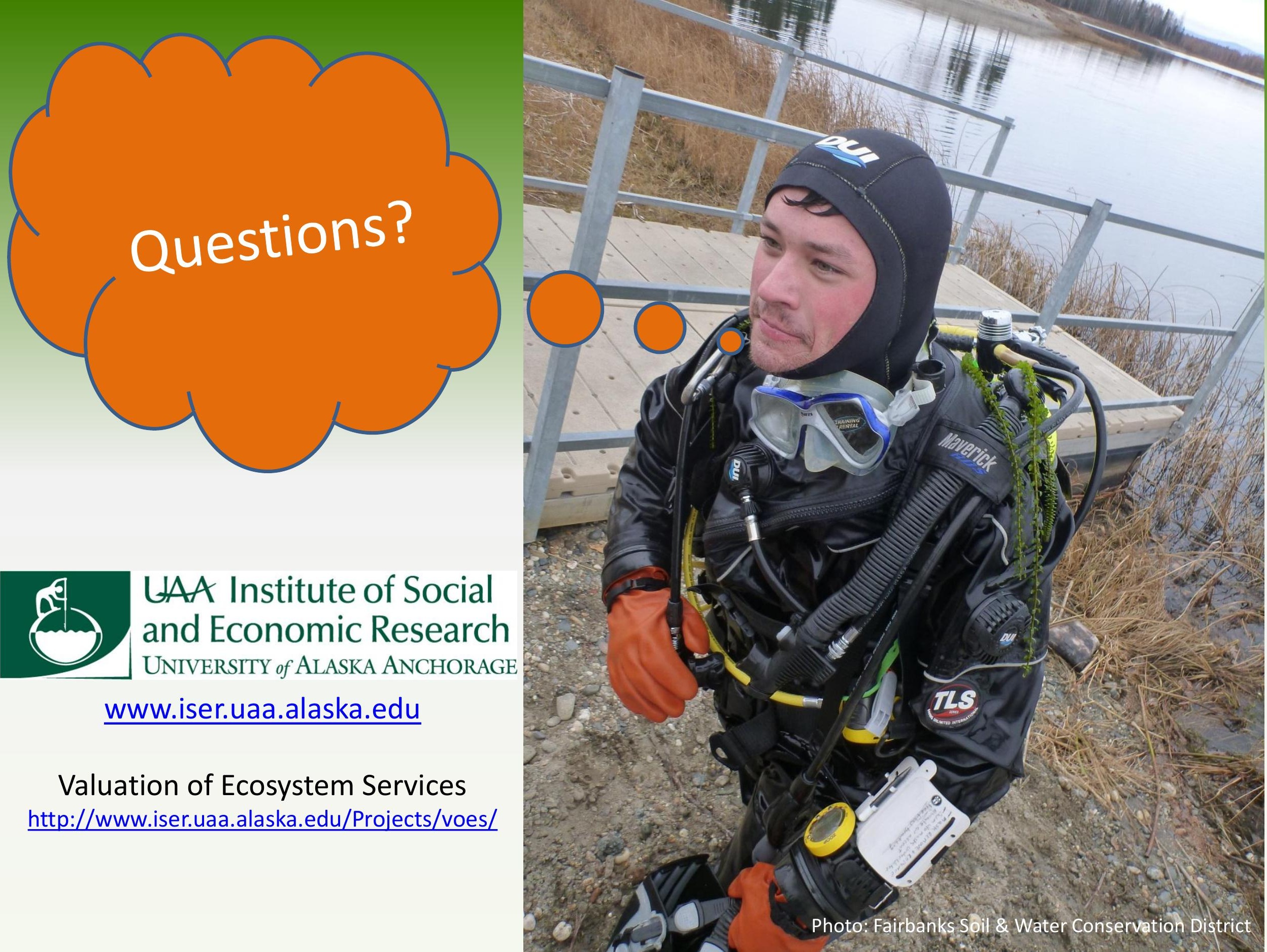

